\section{Atherosclerosis in patients with endometriosis}

Amir Hassan Habibi, ${ }^{1}$ Elham Alizadeh, ${ }^{1}$ Neda Hashemi, ${ }^{1}$ Saeed Razmeh, ${ }^{2}$ Setareh Siah Mansuri, ${ }^{1}$ Zahra Ghurchian, ${ }^{1}$ Amir Hassan Rezaee ${ }^{1}$

${ }^{1}$ Iran University of Medical Sciences, Tehran; ${ }^{2}$ Yasuj University of Medical Sciences, Yasuj, Iran

\begin{abstract}
Endometriosis refers to endometrial tissue implantation including stromal and epithelial tissue outside the uterus. It is an often painful disorder that involves the ovaries and fallopian tubes. The association between endometriosis and atherosclerosis is interesting. The present study was conducted to assess the Doppler findings of the carotid artery in patients with and without endometriosis. In this study that included 30 patients with endometriosis and 30 control subjects, all patients underwent carotid ultrasonography, and the measurement of intima-media thickness (IMT) was done in the distal common carotid artery, proximal to the origin of the carotid bulb, and finally, we compare the IMT in two groups. This study revealed that there are no differences in the IMT between patients with and without Endometriosis.
\end{abstract}

\section{Introduction}

Endometriosis refers to endometrial tissue implantation including stromal and epithelial tissue outside the uterus. The usual signs and symptoms include pelvic pain, dyspareunia, dysmenorrhea, infertility, bladder/bowel symptoms. ${ }^{1,2}$ It affects $5-8 \%$ of adolescents and $8-12 \%$ of women in reproductive age. The most common sites of endometriosis are primarily seen in the pelvic, peritoneum, ovary, and rectovaginal septum. ${ }^{3,4}$ No pathogenesis theory that can explain all the manifestations of endometriosis is still not fully developed, but there are a number of theories including retrograde menstruation, coelomic metaplasia, metastatic spread, and altered immunity. ${ }^{5,6}$ Patients with endometriosis usually have chronic inflammation and systemic oxidative stress and are in a pro atherogenic condition that increases the risk of atherosclerosis in various vessels, including carotid arteries. ${ }^{7-9}$ Accordingly, and in light of the importance of this article, we have reviewed the comparison of Doppler findings of the carotid artery in patients with and without endometriosis.

\section{Materials and Methods}

This cross-sectional study included 30 patients with endometriosis and 30 control subjects, all patients underwent laparoscopy and the diagnosis of endometriosis confirmed with histological examination. The inclusion criteria consisted of women with/without endometriosis between ages 20-50. The exclusion criteria included of diabetes mellitus, hypertension, cardiovascular disease. This study was reviewed and approved by the research ethics committee, and all patients provided informed consent before participation to the study. All patients underwent carotid ultrasonography by a $7-15 \mathrm{MHz}$ linear probe blindly by an expert radiologist. The measurement of intima-media thickness (IMT) was done in the distal common carotid artery, proximal to the origin of the carotid bulb also we assessed the PSV, end-diastolic volume (EDV), and pulsatility index (PI). All analyses have been performed with the SPSS 22 and $\mathrm{P}<0.05$ regarded as a significant level. The differences between the means of these variables were tested by using t-student test.

\section{Results}

Our study included 30 women with endometriosis and 30 matched control. The age range was $15-51$ years. The mean age was 33.1 years. There is no significant difference in the mean age, body mass index (BMI), fasting blood sugar (FBS), triglyceride (TG), cholesterol, High-density lipoproteins (HDL), and Low-density lipoprotein (LDL) between women with and without endometriosis. In patients with endometriosis, the mean IMT was $0.488 \pm$ $0.025 \mathrm{~cm}$ and $0.484 \pm 0.023 \mathrm{~cm}$ in the right and left carotid artery and in control group was $0.422 \pm 0.018 \mathrm{~cm}$ and $0.432 \pm 0.015 \mathrm{~cm}$ in the right and left carotid artery that this difference not significant in two groups. Also, there is no significant difference in EDV, systolic blood pressure (SBP) and PI in two groups (Table 1)

\section{Discussion and Conclusions}

Endometriosis affects $10-15 \%$ of reproductive-aged women. It commonly occurs in the pelvis but also can arise in the abdomen, lungs, central nervous system and musculoskeletal system. The common presentation includes pelvic pain, infertility, dyspareunia and dysmenorrhea. ${ }^{8,10}$ The results of our study show that there is no significant difference in lipid profile in
Correspondence: Saeed Razmeh, Yasuj University of Medical Sciences, Yasui, Iran.

E-mail:srazmeh@yahoo.com

Key words: Endometriosis; Atherosclerosis; Intima-media thickness.

Contributions: AHH, EA contributed to the design and implementation of the research; $\mathrm{NH}$, SR were involved in planning and supervised the work; SSM, ZG, AHR contributed to the analysis of the results and to the writing of the manuscript.

Conflict of interest: the authors declare no potential conflict of interest.

Funding: none

Received for publication: 9 February 2018. Revision received: 4 December 2018.

Accepted for publication: 21 March 2019.

This work is licensed under a Creative Commons Attribution NonCommercial 4.0 License (CC BY-NC 4.0).

CC Copyright A.H. Habibi, et al., 2019

Licensee PAGEPress, Italy

Urogynaecologia 2019; 31:214

doi:10.4081/uij.2019.214

patients with and without endometriosis. Several study assessed the lipid profile in patients with endometriosis in comparison with healthy patients with different results. In a study that evaluated the lipid profile in patients with and without endometriosis found that endometriosis patients had an increased LDL and HDL cholesterol levels compared with controls group. ${ }^{11}$ In another study that done by Almassinokiani et al., show that the patients with endometriosis, had higher total cholesterol level in comparison with the control group but there is not a significant difference in mean level of LDL, HDL and triglyceride in two group. ${ }^{12}$ In a study that done by Pretta et al. assessed the IMT in patients with and without endometriosis, shows that there in not significant difference in IMT in patients with and without endometriosis. ${ }^{13}$ In our study also there is no significant difference in IMT, EDV, SBP and PI in two groups. In a study that done by Santoro et al., assessed the ultrasound evaluation of IMT and flowmediated dilation (FMD) in patients with and without endometriosis. In this study FMD compared with the control group was lower in patients with endometriosis but there is no significant difference in IMT in two groups. ${ }^{14}$ Another study done by Tani et al. shows that women with endometriosis had higher Serum high-sensitivity C-reac- 
Table 1. There is no significant difference in mean age, body mass index, fasting blood sugar, triglyceride, cholesterol, high-density lipoproteins, and low-density lipoprotein, end-diastolic volume, systolic blood pressure and pulsatility index in two groups.

\begin{tabular}{lccc} 
Variables & Patients & Controls & P value \\
Age & 35 & 31 & .95 \\
Body mass index & 25 & 24 & .175 \\
\hline Triglyceride & 124 & 110 & .38 \\
Cholesterol & 139 & 132 & .43 \\
\hline High-density lipoprotein & 36 & 43 & 420 \\
Low-density lipoprotein & 88 & 70 & .18 \\
\hline Intima-media thickness R & .665 & .668 & .068 \\
Intima-media thickness L & .663 & .662 & .056 \\
\hline Fasting blood sugar & 95 & 89 & .075 \\
Systolic blood pressure & 82 & 83 & .025 \\
\hline End-diastolic volume & 32 & 37 & .98 \\
Pulsatility index & 1.1 & .95 & .238 \\
\hline
\end{tabular}

tive protein level and arterial stiffness in compared with controls group. ${ }^{15}$ In our study, the confounding factors including age, BMI, lipid profile were similar in two groups. Moreover, diseases such as diabetes, hypertension that can cause atherosclerosis were excluded. In conclusion, our study suggests that women with endometriosis do not have increased IMT in compared to healthy patients, but more study with more cases and variable should be done in future.

\section{References}

1. Bergqvist A. Different types of extragenital endometriosis: a review. Gynecol Endocrinol 1993;7:207-21.

2. Brosens I, Benagiano G. Endometriosis, a modern syndrome. Indian J Med Res 2011;133:581.

3. Allaire C. Endometriosis and infertility: a review. J Reproduct Med 2006;51: 164-8.

4. Macer ML, Taylor HS. Endometriosis and infertility: a review of the pathogenesis and treatment of endometriosisassociated infertility. Obstetrics Gynecol Clin North Am 2012;39:53549.

5. Burney RO, Giudice LC. Pathogenesis and pathophysiology of endometriosis. Fertil Steril 2012;98:511-9.

6. Mahmood TA, Templeton A. Pathophysiology of mild endometriosis: review of literature. Human Reproduct 1990;5:765-84.

7. Kinugasa S, Shinohara K, Wakatsuki A. Increased asymmetric dimethylarginine and enhanced inflammation are associ- ated with impaired vascular reactivity in women with endometriosis. Atherosclerosis 2011;219:784-8.

8. Nothnick WB. Treating endometriosis as an autoimmune disease. Fertil Steril 2001;76:223-31.

9. Santanam N, Song M, Rong R, et al. Atherosclerosis, oxidation and endometriosis. Free Rad Res 2002;36:1315-21.

10. Sinaii N, Cleary SD, Ballweg M, et al. High rates of autoimmune and endocrine disorders, fibromyalgia, chronic fatigue syndrome and atopic diseases among women with endometriosis: a survey analysis. Human Reproduct 2002;17:2715-24.

11. Melo AS, Rosa-e-Silva JC, de Sá Rosa ACJ, et al. Unfavorable lipid profile in women with endometriosis. Fertility Steril 2010;93:2433-6.

12. Almassinokiani F, Mehdizadehkashi A, Amirkhani J, et al. Comparing the serum lipid profile levels in women suffering from endometriosis with healthy women. J Minimal Invasive Surg Sci 2014;3:e19818.

13. Pretta S, Remorgida V, Abbamonte LH, et al. Atherosclerosis in women with endometriosis. Eur J Obstetr Gynecol Reproduct Biol 2007;132:226-31.

14. Santoro L, D'onofrio F, Campo S, et al. Endothelial dysfunction but not increased carotid intima-media thickness in young European women with endometriosis. Human Reproduct 2012;27:1320-6.

15. Tani A, Yamamoto S, Maegawa M, et al. Arterial stiffness is increased in young women with endometriosis. J Obstetr Gynecol 2015;35:711-5. 